Keywords: Reaction mechanism, D-3-Hydroxybutyrate dehydrogenase, Substrate, Inhibitor

\section{MS9-P5 Structures of the photosensory core module of bacteriophytochrome Agp1 from two crystal forms reveal plasticity of the $\mathrm{Pr}$ state}

Norbert Krauß $\beta^{1,2}$, Soshichiro Nagano ${ }^{2,3}$, Patrick Scheerer ${ }^{4}$, Kristina Zubow ${ }^{2}$, Norbert Michael $^{5}$, Katsuhiko Inomata ${ }^{6}$, Tilman Lamparter $^{1}$

1. Karlsruher Institut für Technologie, Botanisches Institut (Germany)

2. Queen Mary University of London, School of Biological and Chemical Sciences (United Kingdom)

3. Justus-Liebig-Universität Giessen, Institut für Pflanzenphysiologie (Germany)

4. Charité - Universitätsmedizin Berlin, Institut für Medizinische Physik und Biophysik (Germany)

5. Technische Universität Berlin, Institut für Chemie (Germany)

6. Kanazawa University, Division of Material Science (Japan)

email: norbert.krauss@kit.edu

Agp1 is a canonical biliverdin-binding bacteriophytochrome from the soil bacterium Agrobacterium fabrum that acts as a light-regulated histidine kinase and uses the red-light-absorbing Pr form as the dark adapted state [1]. At the level of the tertiary structure, the structural changes that occur during photoconversion from $\mathrm{Pr}$ to the far-red-absorbing $\mathrm{Pfr}$ form are characterised by a transition in the so-called tongue region of the $\mathrm{PHY}$ domain from an antiparallel $\beta$-sheet in $\operatorname{Pr}$ to a partially $\alpha$-helical structure in Pfr and rearrangements within the long $\alpha$-helix that connects the GAF and the PHY domain. Based on those crystal structures of photosensory core modules (PCMs) where the protein subunits are arranged as parallel dimers, suggestions have been made as to how changes in the quaternary structure of the PCM associated with photoconversion lead to modulation of the activity of the histidine kinase output module [2, 3, 4]. Here we present crystal structures of the PCM of Agp1 at $2.70 \AA$ resolution and of a surface-engineered mutant at $1.85 \AA$ resolution in $\operatorname{Pr}$. Whereas in the mutant structure the dimer subunits are found to be in anti-parallel orientation, the wild-type structure contains parallel dimers, which structurally differ significantly from the parallel dimers of the PCMs of other phytochromes in Pr. Comparison of the two Agp1-PCM structures reveals relative orientations between the PAS-GAF bidomain and the PHY domains to be different, due to movement about two hinges in the GAF-PHY connecting $\alpha$-helix and in the tongue region that indicates pronounced structural flexibility which may give rise to a dynamic Pr state even at the level of the quaternary structure. The high resolution of the mutant structure enabled us to detect a sterically strained conformation of the chromophore at pyrrole ring $\mathrm{A}$ and its side chain that we attribute to the tight interaction with Pro461 of the PRxSF motif in the tongue region and the restricted conformational degrees of freedom which are due to the covalent linkage between biliverdin (BV) and Cys20. Based on this observation and on additional biochemical data we discuss the potential reasons for the crucial role which the tongue region plays in Pr-to-Pfr photoconversion.

References:

[1] T. Lamparter et al., Proc. Natl. Acad. Sci. U. S. A. 99, 11628-11633 (2002)

[2] H. Takala et al., Nature 509, 245-248 (2014) 
[3] X.J. Yang et al., Structure 23, 1179-1189 (2015)

[4] E.S. Burgie et al., Structure 24, 448-457 (2016)

\section{Agp1-PCM dimers}
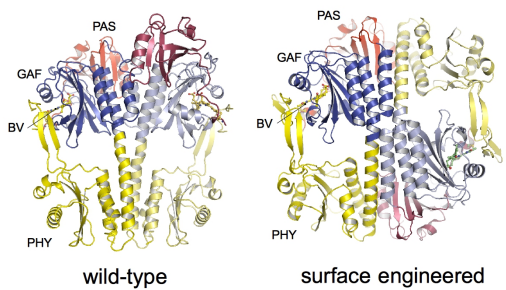

Figure 1. Structures of parallel (left) and anti-parallel dimers (right) of the photosensory core modules (PCMs) of wild-type Agpl and a surface-engineered mutant, respectively. The domains of the PAS-GAF-PHY tridomains are shown in different colours.

Keywords: phytochrome, histidine kinase, dynamic quaternary structure

\section{MS9-P6 Neutron macromolecular crystallography at the FRM II - Or: what can neutrons do for you \\ Andreas Ostermann ${ }^{1}$, Tobias E. Schrader ${ }^{2}$, Michael Monkenbusch ${ }^{3}$, Bernhard Laatsch ${ }^{4}$, Philipp Jüttner ${ }^{1}$, Winfried Petry $^{1}$, Dieter Richter ${ }^{3}$}

1. Heinz Maier-Leibnitz Zentrum (MLZ), Technische Universität München, Lichtenbergstr. 1, 85748 Garching, Germany

2. Jülich Centre for Neutron Science JCNS, Forschungszentrum Jülich GmbH, Outstation at MLZ, Lichtenbergstr.1, 85747 Garching, Germany

3. Institute for Complex Systems ICS, Forschungszentrum Jülich GmbH, 52425 Jülich, Germany

4. Forschungszentrum Jülich GmbH, Engineering and Technology (ZEA-1), 52425 Jülich, Germany

email: Andreas.Ostermann@frm2.tum.de

The research reactor Heinz Maier-Leibnitz (FRM II) is a modern high flux neutron source which feeds at the present 27 state of the art instruments. The newly build neutron single crystal diffractometer BIODIFF is especially designed to collect data from crystals with large unit cells. The main field of application is the structure analysis of proteins, especially the determination of hydrogen atom positions. BIODIFF is a joint project of the Forschungszentrum Jülich (FZJ/JCNS) and the Forschungs-Neutronenquelle Heinz Maier-Leibnitz (FRM II). Typical scientific questions addressed are the determination of protonation states of amino acid side chains in the active center of enzymes and the characterization of the hydrogen bond network between the protein and an inhibitor or substrate. BIODIFF is designed as a monochromatic instrument. By using a highly orientated pyrolytic graphite monochromator (PG002) the diffractometer is able to operate in the wavelength range of $2.4 \AA$ to about $5.6 \AA$. Contaminations of higher order wavelengths are removed by a neutron velocity selector. To cover a large solid angle and thus to minimize the data collection time the main detector of BIODIFF consists of a neutron imaging plate system in a cylindrical geometry. A $\mathrm{Li} / \mathrm{ZnS}$ scintillator CCD camera is available for additional detection abilities. The main advantage of BIODIFF is the possibility to adapt the wavelength to the size of the unit cell of the sample crystal while operating with a clean monochromatic beam that keeps the background level low. BIODFF is equipped with a standard Oxford Cryosystem "Cryostream 700+" which allows measurements in the temperature regime from $90 \mathrm{~K}$ up to $500 \mathrm{~K}$.

Keywords: Protonation states, enzyme function, neutron macromolecular crystallography, water structure 